\title{
A conserved G protein (Drg1p) plays a role in regulation of invasive filamentation in Candida albicans
}

\author{
Xi Chent and Carol A. Kumamoto
}

Correspondence

Carol A. Kumamoto

carol.kumamoto@tufts.edu

Received 25 June 2006

Accepted 5 August 2006

\author{
Department of Molecular Biology and Microbiology, and Genetics Program, Tufts University \\ School of Medicine, 136 Harrison Avenue, Boston, MA 02111, USA
}

\begin{abstract}
During infection, the opportunistic fungal pathogen Candida albicans grows invasively into the tissues of its host, forming filaments that penetrate the host tissue. To search for genes that are important for invasive filamentation, a screen for mutants that were defective in invasion of agar medium was conducted. A mutant carrying an insertion mutation in the locus of a gene, termed here $D R G 1$, was identified. DRG1 encodes a highly conserved cytoplasmic G protein, with orthologues in the genomes of organisms from humans to yeast and archaea. C. albicans strains lacking Drg1p were defective in producing filaments that penetrated agar media, but produced filaments normally under other conditions, such as during liquid growth. When inoculated intravenously into mice, the drg1 null mutant caused delayed lethality accompanied by delayed invasive growth in the kidneys of the host, in comparison with those of the wild-type strain. These results implicate Drg1 $\mathrm{p}$ in the control of invasive filamentation in the laboratory, and in the progression of invasive disease in the host.
\end{abstract}

\section{INTRODUCTION}

Candida albicans is one of the most important opportunistic fungal pathogens in humans. C. albicans causes diseases ranging from mild superficial infections to more severe bloodstream infections that may lead to systemic disease. Examples of superficial infections include oral thrush found in young children, and vaginal yeast infections in women. Systemic infections usually occur in immunocompromised hosts whose immune systems may be suppressed by chemotherapy, weakened by other diseases, or not fully developed.

During infection, C. albicans grows invasively into the tissues of its host. C. albicans is a dimorphic fungus and, during invasive growth, typically assumes a filamentous morphology. Under other conditions, C. albicans is found in a yeast form. The ability to convert from yeast-form cells to filamentous hyphae (elongated cells lacking constrictions at the septa) and pseudohyphae (elongated cells with constrictions at the septa) is believed to be important for invasiveness and virulence (reviewed by Kumamoto \& Vinces, 2005b).

To probe the mechanisms that allow C. albicans to invade host tissue, laboratory studies have made use of $C$. albicans

†Present address: Department of Pediatrics, Stanford University School of Medicine, Palo Alto, CA 94305, USA.

Abbreviations: DAPI, 4',6-diamidino-2-phenylindole; GFP, green fluorescent protein; REMI, restriction-enzyme-mediated integration. invasion into agar matrices. Several proteins, including signal transduction proteins and transcription factors, which regulate invasive growth into agar have been previously described (reviewed by Kumamoto \& Vinces, 2005a). The bHLH transcription factor Efglp is a negative regulator of filamentation when cells are embedded in an agar matrix (Giusani et al., 2002), even though Efglp has a critical positive role in filamentation under most other conditions (Stoldt et al., 1997).

In this study, a search for C. albicans genes that are important for invasive filamentation in agar was undertaken. Mutations affecting the gene, orf19.5083, were found to result in reduced invasiveness. This gene is highly homologous to the RBG1 (ribosome-interacting GTPase)/ FUN11 (function unknown now) gene of Saccharomyces cerevisiae, and to the gene encoding DRG1 (developmentally regulated GTP-binding protein) of Xenopus laevis (Kumar et al., 1993) and other species. The C. albicans gene will be referred to as $D R G 1$ in this paper.

Previous studies have suggested that the function of DRG1 may be related to growth control. For example, ectopic expression of mouse DRG1 enhances oncogenic transformation by c-myc and ras (Mahajan et al., 1996). Mouse DRG1 interacts with an oncogene for stem cell acute lymphoblastic leukaemia, TAL1/SCL1 (Mahajan et al., 1996). TAL1/SCL1 encodes a bHLH transcription factor whose HLH domain interacts with the C-terminal region of DRG1. Thus, DRG1 may influence growth control by 
binding and regulating transcription factors. Expression of DRG1 also correlates with growth; $D R G 1$ is highly expressed during embryonic development in Xenopus (Kumar et al., 1993) and mice (Sazuka et al., 1992), and is expressed at higher levels in growing versus non-growing plant cells (Devitt et al., 1999). Despite these clues, the function of DRG1 is not well understood in any species.

In this study, we showed that a C. albicans $\operatorname{drg} 1$ mutant was attenuated in invasiveness in an agar matrix, but was capable of hyphal growth under other conditions. In addition, the drg1 null mutant exhibited delayed invasiveness in host tissue, and delayed lethality during infection. As invasive growth is a contact-dependent response, these findings suggest that Drg1p functions in contact-dependent signalling or responses.

\section{METHODS}

Strains. C. albicans strain CAI-4 (Dura3:: imm434/ $\Delta$ ura3: : imm434) (Fonzi \& Irwin, 1993) was used to construct all $C$. albicans strains (listed in Table 1).

To construct drg1 null mutants by the Ura-blaster method (Fonzi \& Irwin, 1993), plasmids pXC110 and pXC111, encoding $5^{\prime}$ and $3^{\prime}$ fragments of the DRG1 locus flanking hisG-URA3-hisG, were digested with $K p n I$ and HindIII. Strains were characterized by Southern blotting to verify that all alleles of $D R G 1$ were deleted. For complementation analysis, $\mathrm{Ura}^{-}$derivatives of $\operatorname{drg} 1$ null mutants were integratively transformed with BsaBI-digested pXC155.

For cloning, Escherichia coli strains XL-1 Blue or JM109 were used.

Media and growth conditions. For culturing C. albicans, standard media included YP ( $1 \%$ yeast extract and $2 \%$ bacto peptone) with either $2 \%$ glucose (YPD) or $2 \%$ sucrose (YPS), or CM-Ura (complete minimal medium lacking uridine and uracil) (Ausubel et al., 1989). For solid media, $2 \%$ agar was added. For selection of $\mathrm{Ura}^{-}$strains, CM-Ura medium was supplemented with $60 \mu \mathrm{g}$ uridine $\mathrm{ml}^{-1}$ and $1 \mathrm{mg}$ 5-fluoroorotic acid $\mathrm{ml}^{-1}$ (Kelly et al., 1987). For the colony morphology assay, YPS medium containing $1 \%$ agar (Difco) was used (Brown et al., 1999). Synthetic low ammonium, Lee's, proline and GlcNAc media were prepared as described by Brown et al. (1999).

E. coli strains were cultured in L broth or on L plates (Miller, 1972), with ampicillin added to a concentration of $100 \mu \mathrm{g} \mathrm{ml}^{-1}$.

Construction of plasmids. Synthetic oligonucleotides are described in Table 2. pXC110 and pXC111 were constructed from p510 (http://www.sacs.ucsf.edu/home/JohnsonLab/burk/Candisrupt. html) (kind gift of B. Braun and A. Johnson, University of California, San Francisco). For pXC110, the 5' DRG1 fragment was amplified from chromosomal DNA with primers XC-01 and XC-02. The DRG 3' fragment was amplified with primers XC-03 and XC04. pXC111, used to delete the last allele of DRG1, contained DRG1 sequences that were within the sequences deleted by pXC110. For

Table 1. Fungal strains used in this study

\begin{tabular}{|c|c|c|}
\hline Strain & Genotype & Source or reference \\
\hline \multicolumn{3}{|l|}{ C. albicans } \\
\hline CAI-4 & SC5314 ura3/ura3 & Fonzi \& Irwin (1993) \\
\hline CKY101 & CAI-4 [ade2::pDBI52 (URA3)] & Brown et al. (1999) \\
\hline CKY136 & CAI-4 efg1:: his/efg1:: his [ade2::pDBI52 (URA3)] & Giusani et al. (2002) \\
\hline HLC74 & CAI-4 efg1:: his/efg1:: his [leu2::HLB134 (EFG1)] & Lo et al. (1997) \\
\hline XC101 & CAI-4, [leu2::pRC3915 (URA3)] (two copies of chromosome 1) & Chen et al. (2004) \\
\hline $\mathrm{XC121}$ & CAI-4, [ade2::pXC154 (URA3)] (two copies of chromosome 1) & This study \\
\hline $\mathrm{XC} 210$ & Insertion of pXC132 into the chromosome of CAI- 4 & This study \\
\hline XC211 & Insertion of pXC132 into the chromosome of CAI- 4 & This study \\
\hline XC301 & CAI-4, drg1 $1 \Delta:$ his-URA3-his/DRG1/DRG1 & This study \\
\hline XC302 & CAI-4, $\operatorname{drg} 1 \Delta::$ his/DRG1/DRG1 & This study \\
\hline XC303 & CAI-4, $\operatorname{drg} 1 \Delta::$ his/drg1 $1 \Delta:$ his-URA3-his/DRG1 & This study \\
\hline XC304 & CAI-4, $\operatorname{drg} 1 \Delta::$ his/drg1 $1 \Delta:$ his/DRG1 & This study \\
\hline XC305 & 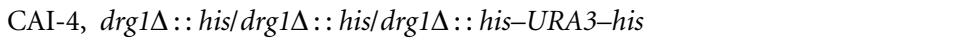 & This study \\
\hline XC306 & CAI-4, $\operatorname{drg} 1 \Delta::$ his/drg1 $1 \Delta::$ his/drg1 $1 \Delta:$ his & This study \\
\hline XC308 & CAI-4, $\operatorname{drg} 1 \Delta::$ his/ $\operatorname{drg} 1 \Delta::$ his (two copies of chromosome 1 ) & This study \\
\hline $\mathrm{XC} 413$ & XC308 $\left[l e u 2:: p R C 3915\left(U R A 3^{+}\right)\right]$ & This study \\
\hline XC414 & $\mathrm{XC} 308\left[\operatorname{drg} 1:: \mathrm{pXC155}\left(U R A 3^{+}, D R G 1^{+}\right)\right]$ & This study \\
\hline XC415 & XC308 [ade2::pXC154 $\left(\right.$ URA3 $\left.\left.^{+}\right)\right]$ & This study \\
\hline XC416 & $\mathrm{XC} 308\left[\operatorname{drg} 1:: \mathrm{pXC156}\left(U R A 3^{+}, m y c-D R G 1\right)\right]$ & This study \\
\hline XC310 & 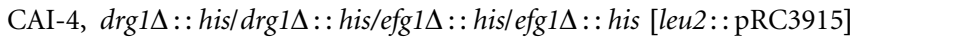 & This study \\
\hline XC311 & 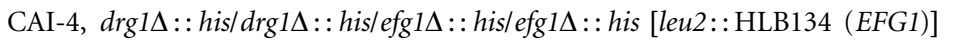 & This study \\
\hline \multicolumn{3}{|c|}{ S. cerevisiae } \\
\hline EGY40 & MAT $\alpha$ ura3-1 his3-11 trp1-1 leu2-3,112 & Golemis et al. (1996) \\
\hline
\end{tabular}


Table 2. Synthetic oligonucleotides used in this study

\begin{tabular}{|c|c|}
\hline Name & Sequence $\left(5^{\prime}-3^{\prime}\right)^{*}$ \\
\hline XC-01 & cccAAGCTTttcattgagagtgtggtaagt (HindIII) \\
\hline XC-02 & aaCTGCAGtgctagccetttgaagtaatc (PstI) \\
\hline XC-03 & gcGAGCTCaatgtcaagtagatccaaaatatg (SacI) \\
\hline XC-04 & gtgtatGGTACCtcggttaagca $(K p n \mathrm{I})$ \\
\hline XC-05 & cccAAGCTTactaaatcatgactaccgtgg (HindIII) \\
\hline XC-06 & aaCTGCAGcagcttctgaatgggttcct $(P s t \mathrm{I})$ \\
\hline $\mathrm{XC}-07$ & gcGAGCTC $\overline{c c a c c a a a g a c t t g t g a a t a g}(\mathrm{~S} a \mathrm{I})$ \\
\hline XC-08 & cggGGTACCtcgttctctattgaagagttga $(K p n \mathrm{I})$ \\
\hline XC-09 & cGAGCTCggtccagttctggtcgagtga (SacI) \\
\hline $\mathrm{XC}-10$ & ccTTAATTAAggttcagcttgtggttgatcttg $(\mathrm{PacI})$ \\
\hline $\mathrm{XC}-11$ & gattacttcaaaggGCTAGCa (NheI) \\
\hline $\mathrm{XC}-12$ & ttaattaacccggggatccgcatgatttagtgggttctttt \\
\hline $\mathrm{XC}-13$ & cggatccccgggttaattaa \\
\hline $\mathrm{XC}-14$ & tttctccacggtagtcatgtagtgattgat \\
\hline $\mathrm{XC}-15$ & gttGGATCCtttcttcaaaatcgtaatcacat $($ Bam HI) \\
\hline $\mathrm{XC}-16$ & ggtGGATCC $t c t t c a a a a t c g t a a t c a c a t c t(B a m H I)$ \\
\hline $\mathrm{XC}-17$ & ggtGAATTCaagaacccactaaatcatgact (EcoRI) \\
\hline $\mathrm{XC}-18$ & cgGAATTCtcaacgtattctataccctattac (EcoRI) \\
\hline XC-19 & 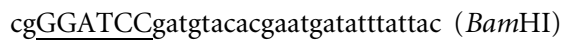 \\
\hline XC-20 & ttgacaggagccttagatgga \\
\hline $\mathrm{XC}-21$ & agaagaagaacagcgtaggtt \\
\hline $\mathrm{XC}-22$ & caacacatagattgcaggaatg \\
\hline XC-23 & tcactacattgacaacagtcc \\
\hline XC-24 & cgGAATTCgatgctacttacggtaaattga (EcoRI) \\
\hline XC-25 & aaCTGCAGgatatcgtcgacatgcatgga $(P s t \mathrm{I})$ \\
\hline
\end{tabular}

${ }^{\star}$ Restriction sites (listed in parentheses) introduced into primer sequences for cloning purposes are shown in upper-case type.

construction of pXC111, a DRG1 5' fragment was amplified using primers XC-05 and XC-06, and a $3^{\prime}$ fragment was amplified using primers XC-07 and XC-08.

pXC155 $\left(D R G 1^{+}\right)$and pXC154 (empty vector) were used for

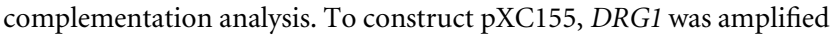
by PCR using primers XC-09 and XC-10. After digestion with SacI and PacI, the fragment was ligated into SacI/PacI-digested pNUB1 (pNEB193 carrying the C. albicans XbaI to ScaI URA3 fragment cloned into the NdeI site). pXC154 (vector-only control) was generated by deleting the maltase promoter from pDBI52, and carried the $C$. albicans URA3 gene and a fragment of C. albicans ADE2. For construction of pXC156, encoding a myc-tagged DRG1 allele, PCR fragments were generated using primers $\mathrm{XC}-11$ and $\mathrm{XC}-12$ with pXC155 $\left(D R G 1^{+}\right)$as a template, and using primers XC-13 and XC-14 with pFA6a-13Myc-kanMX6 (Longtine et al., 1998) as a template. These two PCR fragments were mixed and amplified with primers XC11 and $\mathrm{XC}-14$, generating a fragment that contained part of the DRG1 promoter, $13 \mathrm{Myc}$, and part of the DRG1 ORF. This fragment was digested with NheI and BtgI, and ligated into NheI/BtgI-digested pXC155 $\left(D R G 1^{+}\right)$, generating pXC156. For transformation, pXC156 was digested with $B s a \mathrm{BI}$.

lexA-DRG1 was constructed by PCR amplification of the DRG1 gene with primers XC-15 and XC-16, and cloning of the PCR product into vector pEG202 (Golemis et al., 1996). DRG1-lexA was constructed by PCR amplification of the DRG1 gene with primers XC-17 and XC-16, and cloning of the PCR product into vector pNLexA (Golemis et al.,
1996). GAL4AD-EFG1 was constructed by PCR amplification of $E F G 1$ with primers XC-18 and XC-19, and cloning of the PCR product into vector pGAD-C2 (Giusani et al., 2002; James et al., 1996). plexA-Fos and pC1.1.2, encoding GAL4AD-SLK19 (control fusion proteins that are not expected to interact with either Efglp or Drglp), were generously provided by R. Brent (Massachusetts General Hospital, Boston) and R. Kamieniecki (Tufts University, Boston).

pXC132, containing BamHI, NsiI, SalI and EcoRV sites upstream of a promoterless $y E G F P$ gene that was fused to the $3^{\prime}$ UTR of ACT1 in a pNUB1 backbone, was used for insertional mutagenesis.

DNA isolation and analysis. PCR, restriction digestion and gel electrophoresis were performed by standard methods, as described elsewhere (Sambrook et al., 1989). Automated DNA sequencing was performed by Michael Berne and coworkers at the Tufts University Core Facility. Chromosomal DNA isolation and Southern blot hybridization were performed by standard methods, as described previously (Burke et al., 2000; Sambrook et al., 1989). DNA probes were labelled with $\left[\alpha-{ }^{32} \mathrm{P}\right] \mathrm{dATP}$ by random priming.

To demonstrate that the DRG1 gene had been deleted, a probe corresponding to a sequence upstream of the DRG1 ORF was generated by PCR using primers XC-20 and XC-21, and was used to probe a Southern blot of MslI-digested chromosomal DNA from candidate strains. To verify that all copies of DRG1 had been deleted, a second probe, corresponding to sequences within the DRG1 ORF, was generated by PCR using primers XC-22 and XC-23, and used to probe the same blot.

For inverse PCR, genomic DNA from strain XC210 was digested with RsaI, HaeI or AluI separately. Following ligation, the circular DNA products were used as templates for PCRs using primers XC-24 and XC-25 (homologous to sequences within pXC132). The PCR products were purified from a $1 \%$ agarose gel, and the DNA was sequenced using the primers described above.

Electroporation and restriction-enzyme-mediated integration (REMI) of C. albicans. The method used was that described by Brown et al. (1996), with minor modifications. Cells were grown to $\mathrm{OD}_{600} \sim 0 \cdot 5-0 \cdot 6$, harvested, and treated with $0 \cdot 1 \mathrm{M}$ lithium acetate, $0.01 \mathrm{M}$ Tris $/ \mathrm{HCl}, \mathrm{pH} 7 \cdot 5$, and $1 \mathrm{mM}$ EDTA for $45 \mathrm{~min}$ at $30{ }^{\circ} \mathrm{C}$. DTT was added to a concentration of $2 \mathrm{mM}$, and the cells were incubated for an additional $15 \mathrm{~min}$ at $30^{\circ} \mathrm{C}$. After washing with cold water, and with cold $10 \%(\mathrm{v} / \mathrm{v})$ glycerol, cells were resuspended in a small volume of ice-cold $10 \%$ glycerol. For REMI, $20 \mu \mathrm{g}$ plasmid DNA was digested with BamHI and then precipitated using ethanol. One hundred units of BamHI mixed with the digested DNA were added to $40 \mu$ concentrated strain CAI- 4 cells kept on ice. The mixture of cells and DNA was electroporated at $1.5 \mathrm{kV}$, $200 \Omega$ and $25 \mu \mathrm{F}$, and plated on CM-Ura. For homologous recombination, $10 \mu \mathrm{g}$ digested plasmid DNA was added to $40 \mu \mathrm{l}$ concentrated cells, and electroporated as above.

Analysis of filamentation. For analysis of filamentation under embedded conditions, actively growing cells in YPD medium ( $\sim 400$ c.f.u.) were mixed with molten YPS agar, and plated. Plates were incubated for several days. At various times, 100-200 embedded colonies were examined, and the percentage of filamentous colonies was determined (a colony that contained at least 20 filaments protruding from the periphery of the colony was arbitrarily defined as a filamentous colony). Colonies were photographed with a Nikon E400 microscope at $\times 4$ magnification.

To study filamentous growth in $10 \%$ serum and Lee's, proline and GlcNAc media, strains were grown in CM-Ura liquid medium at room 
temperature, washed, starved in water, and added to each medium. After incubation at $37^{\circ} \mathrm{C}$ for $6 \mathrm{~h}$, cells were observed microscopically, and the percentage of filamentous cells was determined.

Protein localization. Immunofluorescence methods were based on standard methods used for S. cerevisiae (Pringle et al., 1991), with modifications. Briefly, C. albicans cells expressing N-terminally myctagged Drglp were grown in liquid medium, or were embedded in agarose. Cells were fixed in formaldehyde $(4 \cdot 4 \%)$ for $2 \mathrm{~h}$. After fixation, embedded cells were released from the agarose by crushing. After zymolyase treatment, cells were added to wells of polylysinecoated slides, and processed for immunofluorescence with anti-myc antibody clone 4A6 (Upstate), using standard methods. Cells were stained with 4',6-diamidino-2-phenylindole (DAPI), observed by fluorescence microscopy, and photographed using the $\times 60$ objective.

Intravenous inoculation of mice. C. albicans cells were grown for $24 \mathrm{~h}$, and harvested by centrifugation. The cells were washed three times with PBS, counted, and adjusted to a density of $5 \times 10^{6}$ cells $\mathrm{ml}^{-1}$; and $0 \cdot 2 \mathrm{ml}$ of the cell suspension was injected into the lateral tail vein of female CF1 mice (18-20 g body weight; Charles River Laboratories). In each experiment, four mice were inoculated per strain, and this experiment was repeated three times. Mice were observed twice daily after infection with C. albicans. In two experiments, some animals were sacrificed, and their kidneys were dissected, fixed in buffered formalin, sectioned, and stained with haematoxylin and eosin or Gomori methenamine silver.

Two-hybrid analysis. S. cerevisiae strain EGY40 carrying the lacZ reporter plasmid pSH18-34 (Golemis et al., 1996) was transformed with the lexA plasmids pEG202, plexA-DRG1, pNLexA, pDRG1-lexA and plexA-Fos, and GAL4AD plasmids pGAD-C2, pKLEF4 and pC1.1.2, using standard methods (Ausubel et al., 1989). For assays of $\beta$-galactosidase activity, cells grown to exponential phase in synthetic complete medium lacking uracil, histidine and leucine were harvested, and $\beta$-galactosidase activity was determined using ONPG as a substrate, as described elsewhere (Clontech, 2001). Analyses were performed in triplicate.

\section{RESULTS}

\section{An insertion mutation results in defective filamentation under embedded conditions}

In this study, random heterozygous insertion mutations were generated using REMI, as described in Methods. From the first 579 REMI transformants, two interesting strains, termed XC210 and XC211, that exhibited defects in invasive filamentation were identified. These two mutants came from the same REMI pool, which was composed of 44 transformants. Southern blot analysis of the location of the construct indicated that the two strains were probably siblings (data not shown).

Following embedding in YPS agar, and growth at $25^{\circ} \mathrm{C}$, the mutant strains XC210 and XC211 were strongly defective in filamentous growth, in comparison to wild-type strain CKY101 (CAI-4, Ura ${ }^{+}$) (data not shown). Despite the strong defect in filamentous growth under matrixembedded conditions, no obvious defect in growth rate was observed when strains XC210, XC211 and CKY101 were grown in YPD liquid medium, and the defect in filamentous growth was not observed when strains XC210 and XC211 were grown in $10 \%$ serum, or in Lee's, proline or GlcNac medium at $37^{\circ} \mathrm{C}$ (data not shown). Together, these results suggest that the gene disrupted by insertion of pXC132 in strains XC210 and XC211 plays a role in filamentous growth specifically under matrix-embedded conditions.

\section{Strain XC210 contains an insertion in the DRG1 locus}

To find the location of the insertion site in strain XC210, inverse PCR and DNA sequencing were performed, as described in Methods. The inserted DNA construct was found to be located $80 \mathrm{bp}$ upstream of the ORF orf19.5083. This ORF is very similar to those termed DRG1 in other eukaryotic species; therefore, we termed the C. albicans gene DRG1.

In C. albicans, DRG1 encoded a protein of 368 aa with characteristic G1-G5 motifs of GTP-binding proteins (Fig. 1). Outside of these motifs, Drg1p is not very similar to the existing subfamilies of GTP-binding proteins, such as heterotrimeric $G$ proteins or ras-like $G$ proteins. As suggested by Sazuka et al. (1992), Drg1p represents a novel class of GTP-binding proteins.

\section{drg1 null mutants are defective in filamentation under embedded conditions}

To study the function of DRG1, drg1 null mutants were constructed (Fig. 2). The starting strain, CAI-4, was trisomic for chromosome 1, and DRG1 mapped to chromosome 1 . Therefore, the initial heterozygous mutants contained one deleted copy of DRG1 and two intact copies (e.g. XC301 and XC302; Fig. 2). The second-round heterozygous mutants, e.g. XC303 and XC304, contained two deleted copies of DRG1 and one intact copy (Fig. 2). Both types of heterozygous mutants had no obvious defect in filamentous growth when they were grown embedded in YPS agar at $25^{\circ} \mathrm{C}$ (Fig. 3). Since the original insertion mutants were heterozygous for the insertion, these strains probably carried additional mutations.

Five null mutants constructed from two heterozygotes were characterized; some were trisomic (XC306; Fig. 2), and others had lost one copy of DRG1 (XC308; Fig. 2). To demonstrate that the phenotypes of these mutants reflected the absence of $D R G 1$, an intact $D R G 1$ gene under the control of its native promoter was transformed into each of the null mutants (XC414; Fig. 2). The phenotype of XC415, a $d r g 1$ null mutant carrying two copies of the $d r g 1$ locus, is shown in Fig. 3. The drg1 null mutant, carrying URA3 either at its native locus, at $A D E 2$, or at $L E U 2$, was defective in filamentation under embedded conditions at $30^{\circ} \mathrm{C}$ (Fig. 3) and at $25^{\circ} \mathrm{C}$ (data not shown). The filamentous growth defect of the drg1 null mutant could be partially complemented by reintroduction of the $D R G 1^{+}$gene at its native locus, under the control of its native promoter (Fig. 3). A representative colony of each strain grown embedded in YPS agar is shown in Fig. 3(B-D). The drg1/ 


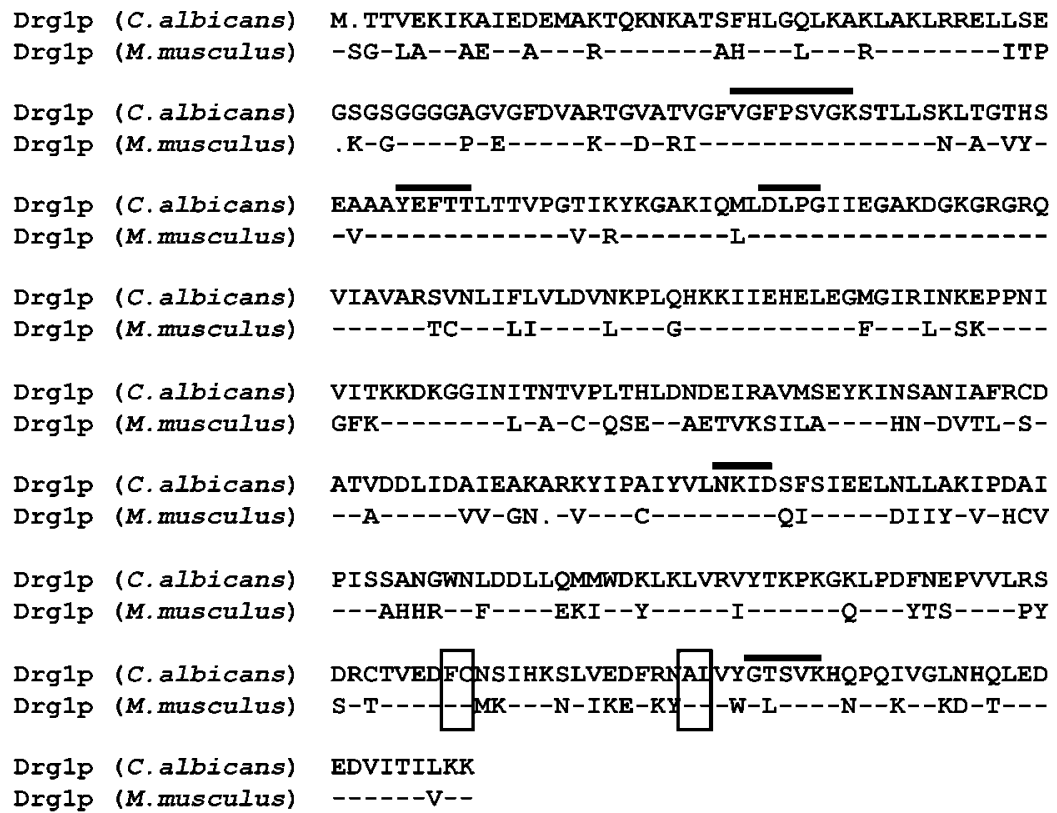

Fig. 1. C. albicans Drg1p. Alignment of C. albicans Drg1p and mouse (Mus musculus) DRG1 is shown. Dashes indicate identical amino acids and dots indicate spaces. Solid lines above sequences indicate the characteristic $\mathrm{G} 1$ to $\mathrm{G} 5$ motifs of $\mathrm{G}$ proteins (Schenker et al., 1994). Boxed residues are important for binding murine DRG1 to the bHLH transcription factor TAL1. drg1/drg1 homozygous null mutants also exhibited a delay in filamentous colony formation under matrix-embedded conditions, and these defects were partially complemented by addition of wild-type $D R G 1^{+}$(data not shown). When the growth rates of the $d r g 1$ null mutants were compared with those of the wild-type strains during growth in YPS + uridine liquid medium, there were no growth defects for any of the $d r g 1$ mutants (data not shown). These results
(A)

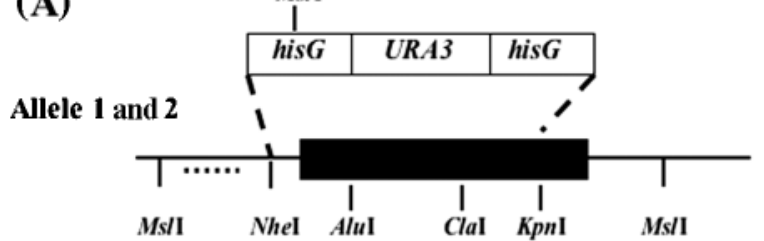

Allele 3

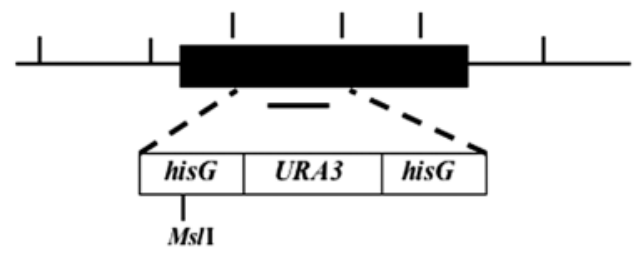

(B)

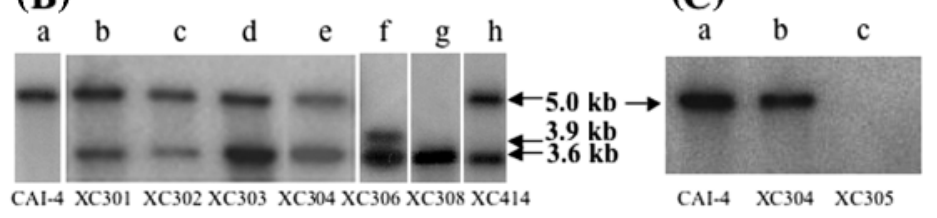

Fig. 2. Southern blot analysis of drg1 null mutants. (A) Restriction map of the DRG1 locus (not drawn to scale). Horizontal lines depict chromosomal DNA, and black boxes indicate the DRG1 ORF. Dashed lines delimit the sequences that were deleted and replaced by the hisG-URA3-hisG URAblaster construct. The dotted line indicates the position of the upstream probe used for Southern blotting. The short line between the Alul and Clal sites indicates the position of the ORF probe used for Southern blotting. (B) Southern blot of Ms/l-digested chromosomal DNA probed with the upstream probe. Positions of the wild-type $D R G 1$ allele $(5.0 \mathrm{~kb})$ and the two drg1D: : hisG-URA3-hisG alleles (3.6 and $3.9 \mathrm{~kb})$ are indicated on the right. XC306 is trisomic, and $\mathrm{XC308}$ has lost the allele that gives the $3.9 \mathrm{~kb}$ band. The two bands in the XC414 sample exhibited equal intensity, consistent with two copies of the DRG1 locus. Lanes: a, CAl-4; b, XC301; c, XC302; d, XC303; e, XC304; f, XC306; g, XC308; h, XC414. (C) Southern blot of Ms/l-digested chromosomal DNA probed with the ORF probe. In lane c, the null mutant completely lacked the DRG1 ORF and failed to yield a band. Lanes: a, CAl-4; b, XC304; c, XC305. 

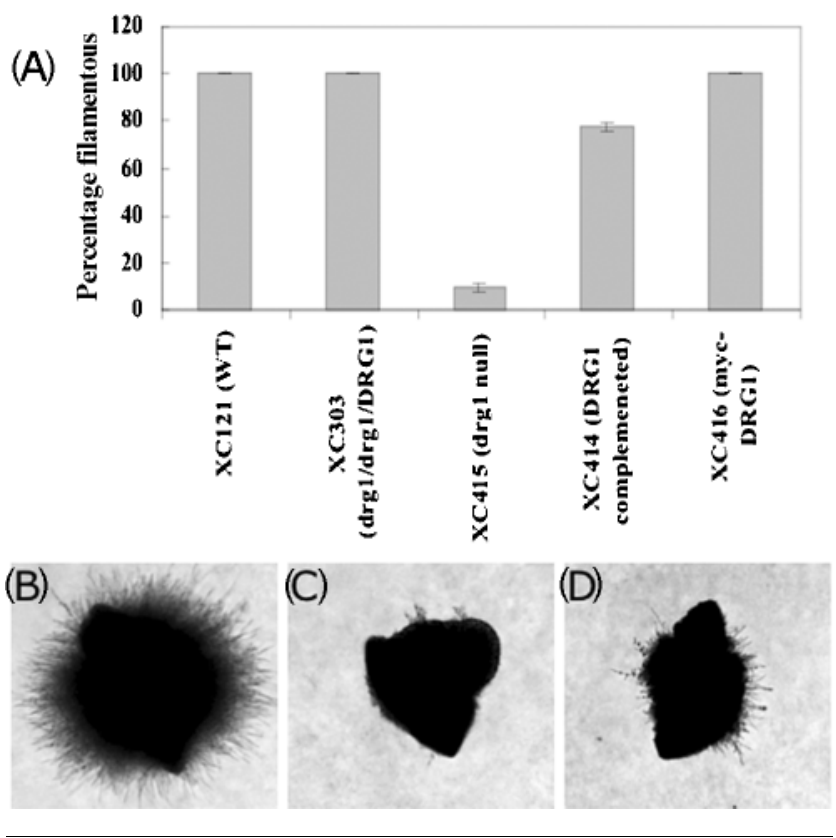

Fig. 3. Defective invasive filamentation in agar medium. Cells were grown embedded in agar medium as described in Methods. (A) After 1 day of growth at $30^{\circ} \mathrm{C}, 100-200$ colonies were examined, and the percentage of filamentous colonies was determined as described in Methods. (B-D) Representative colonies: (B) XC121 (WT), (C) XC415 (drg1 null) and (D) XC414 (DRG1 complemented strain).

support the notion that $D R G 1$ is important for filamentous growth when $C$. albicans is embedded in a matrix.

Filamentous growth of $d r g 1$ null mutants containing two or three copies of chromosome 1 was further tested under different conditions known to induce C. albicans filamentation. The drg1 null mutant was defective in filamentous growth when embedded in different media (e.g. YPS, Spider or low ammonium minimal), and grown at either 25 or $30^{\circ} \mathrm{C}$. At $37^{\circ} \mathrm{C}, \operatorname{drg} 1$ null mutants exhibited defects in filamentation when embedded in low ammonium minimal medium, but not in other media. However, defects in filamentous growth were not observed at $37^{\circ} \mathrm{C}$ when cells were grown on the surface of agar media (YPS, Spider, low ammonium minimal), or grown in liquid media (serum, Lee's, proline or GlcNAc). Therefore, these results indicate that DRG1 is not needed to promote filamentous growth under all conditions.

\section{myc-tagged Drg1p was primarily localized in the cytoplasm}

To study the localization of Drg1p in C. albicans, pXC156, encoding an N-terminally myc-tagged derivative of $D R G 1$, was used. The derivatized gene was recombined into the genome of strain XC308 (drg1 null mutant) at its native locus under the control of its native promoter, as described in Methods. The derivatized mutant gene was biologically
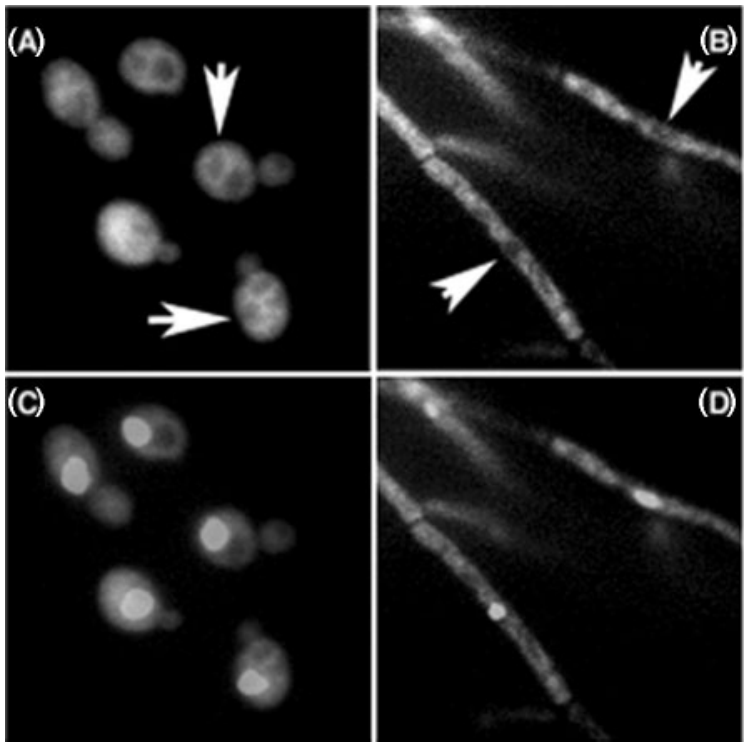

Fig. 4. Immunolocalization of myc-tagged DRG1p. XC416 encoding myc-DRG1 was grown in either liquid medium $(A, C)$ or embedded in agarose $(B, D)$. Cells were fixed and processed for immunofluorescence as described in Methods. ( $A$, $B)$ Localization of myc-tagged Drg1 $p$ visualized with anti-myc clone $4 A 6$ and anti-mouse IgG conjugated to Texas red. (C, D) Overlay of DAPI-stained nuclei and Texas red-stained mycDrg1p. Arrows indicate positions of DAPI-stained nuclei, which exhibit reduced staining for myc-Drg1 $\mathrm{p}$.

active and complemented the $d r g 1$ null mutation (Fig. 3). In contrast, a C-terminally green fluorescent protein (GFP)tagged $D R G 1$ derivative failed to complement the $d r g 1 / d r g 1$ mutation (data not shown); suggesting that modification of the $\mathrm{C}$ terminus is not compatible with this function.

Cells expressing myc-DRG1 or untagged $D R G 1$ were grown in liquid medium, or were embedded in agarose. These cells were fixed and processed for indirect immunofluorescence with anti-myc clone 4A6 (Upstate) and Texas redconjugated anti-mouse IgG secondary antibody. As shown in Fig. 4, most of the cells exhibited perinuclear and cytoplasmic staining. In the majority of cells, the DAPIstained nuclei were deficient in myc-Drglp staining. When cells expressing untagged $D R G 1$ were stained, no fluorescent signal was observed (data not shown). Therefore, we conclude that Drg1p exhibits a predominantly cytoplasmic localization, consistent with the results of studies of murine and human DRG1 (Sazuka et al., 1992; Sommer et al., 1994).

Analysis of cells carrying a transcriptional fusion between the DRG1 promoter region and the gene encoding yEGFP showed that expression of the GFP reporter was similar when cells were grown as yeast cells in liquid medium, or when cells formed embedded filaments (data not shown). Therefore, expression from the DRG1 promoter did not differ strongly between yeast cells and embedded hyphae. 


\section{The drg1 null mutant caused delayed lethality and exhibited reduced invasiveness following intravenous inoculation of mice}

The $d r g 1$ null mutant strain XC413, containing two copies of chromosome 1, was used to test the effect of a $\operatorname{drg} 1$ deletion on the virulence of C. albicans. Strains XC101, XC413 (drg1 null) and XC414 (DRG1 complemented mutant) were intravenously inoculated into mice, as described in Methods. On day 7, nine of 10 mice inoculated with strain XC413 ( $\operatorname{drg} 1$ null mutant) survived, as opposed to four of 12 mice inoculated with strain XC101 (WT), or seven of 11 mice inoculated with XC414 $\left(D R G 1^{+}\right.$complemented). Fisher's exact test showed that the mutant was significantly different from the wild-type strain $(P=0 \cdot 01)$. At later time points, mice inoculated with the $d r g 1$ null mutant succumbed to infection (Fig. 5A); therefore, the mutant strain exhibited delayed lethality. The location of the URA3 gene in XC414 (DRG1 ${ }^{+}$complemented strain) differed from its location in XC101 (WT), and may have influenced the virulence of the complemented strain, as observed previously (Brand et al., 2004; Lay et al., 1998).

To determine whether the $d r g 1$ null mutant exhibited reduced invasiveness, surviving intravenously inoculated mice were sacrificed at 7 days post-inoculation, and their kidneys were examined histologically. As shown in Fig. 5(B), in mice that survived to day 7 , strain XC101 $\left(D R G 1^{+} / D R G 1^{+}\right)$exhibited extensive invasive growth in the renal papilla. Also, the kidney cortex showed evidence of interstitial inflammation with some damage to tubules, but organisms were not observed (data not shown). In contrast, when kidneys of mice inoculated with the $d r g 1$ null mutant were examined, organisms were not observed (Fig. 5C). Kidneys of mice inoculated with the complemented strain resembled those of mice inoculated with wild-type organisms (Fig. 5D). These results show that at an early time point following intravenous inoculation of a host, the $d r g 1$ null mutant exhibited reduced invasiveness.

\section{In the absence of Efg1p, a repressor of embedded filamentation, the drg1 null mutant phenotype was not observed}

Human and mouse DRG1 proteins have been shown to bind bHLH transcription factors via their C-terminal regions. The C-terminal region of C. albicans Drg1p contains the residues that are required for binding of murine DRG1 to TAL1/SCL1 (Fig. 1). Therefore, because the bHLH transcription factor Efglp is an important negative regulator of filamentation during growth under embedded conditions (Giusani et al., 2002), interaction between C. albicans Drg1p and Efglp was analysed using the two-hybrid system. A lexA-DRG1 fusion was tested for interaction with a GAL4AD-EFG1 fusion. As shown in Table 3, while control strains exhibited low levels of the reporter $\beta$-galactosidase, strains containing both $D R G 1$ and EFG1 fusions exhibited approximately two- to threefold higher activity. In contrast, C-terminally tagged DRG1-lexA failed to exhibit
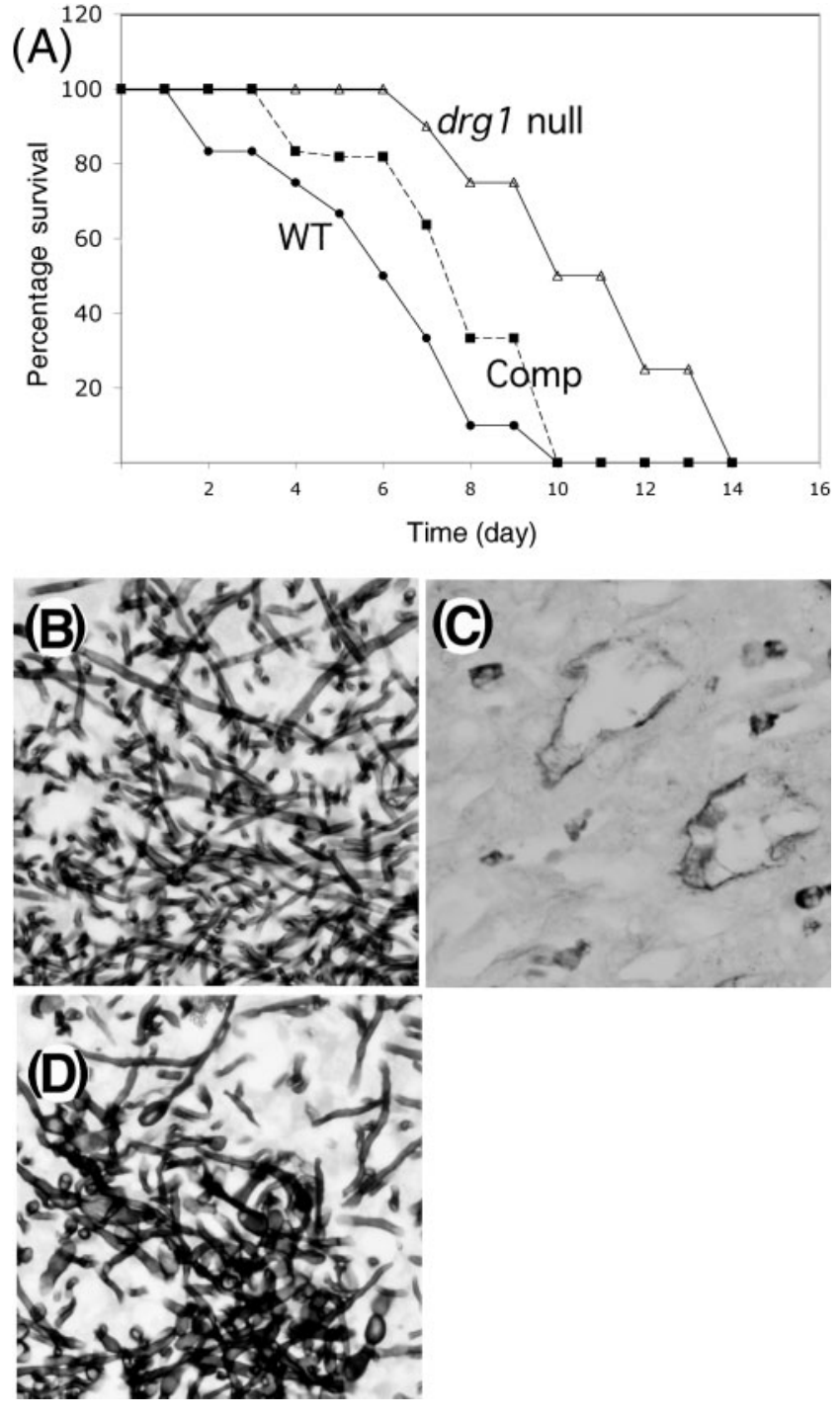

Fig. 5. Delayed lethality caused by the $d r g 1$ null mutant. (A) Cells of strains XC101 (DRG1/DRG1) (•, WT), XC413

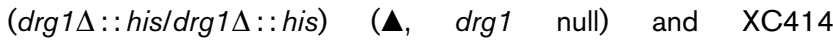

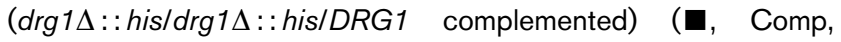
dotted line) were inoculated intravenously into 10-12 mice, and survival as a function of time post-inoculation was monitored. Some animals were sacrificed for histological analysis of kidneys. All mice from three experiments are shown in the graph. (B-D) Mice surviving at day 7 were sacrificed, and kidneys were fixed, sectioned and stained with Gomori methenamine silver staining. Sections were photographed with the $\times 40$ objective. (B) Extensive growth of strain XC101 (WT) in the renal papilla is shown. (C) Organisms were not detected in the renal papilla of mice inoculated with $d r g 1$ null strain $\mathrm{XC413}$. (D) Complemented mutant XC414 exhibiting growth in the renal papilla.

a two-hybrid interaction with GAL4AD-EFG1 (Table 3), consistent with the notion that modification of the $\mathrm{C}$ terminus of Drglp is incompatible with its function. Therefore, Drglp and Efglp may interact, and the effects 
Table 3. Two-hybrid interaction between DRG1 and EFG1

NA, Not applicable.

\begin{tabular}{|c|c|c|c|c|}
\hline Sample & $\operatorname{lex} A$ fusion & GALAAD fusion & Activity ${ }^{\star}$ & $P$ value $\dagger$ \\
\hline 1 & $\operatorname{lexA} A-D R G 1$ & GAL4AD-EFG1 & $8 \cdot 9 \pm 1 \cdot 0$ & NA \\
\hline 2 & Unfused $\neq$ & GAL4AD-EFG1 & $3 \cdot 8 \pm 1 \cdot 4$ & $0 \cdot 001$ \\
\hline 3 & $\operatorname{lexA} A R G 1$ & Unfused $\ddagger$ & $3 \cdot 5 \pm 0 \cdot 7$ & $0 \cdot 006$ \\
\hline 4 & $\operatorname{lex} A-D R G 1$ & GAL4AD-SLK19S & $3 \cdot 7 \pm 1 \cdot 1$ & $0 \cdot 004$ \\
\hline 5 & $\operatorname{lex} A-f o s \S$ & $G A L 4 A D-E F G 1$ & $3 \cdot 0 \pm 1 \cdot 0$ & $0 \cdot 002$ \\
\hline 6 & PNlexA (unfused) $\ddagger$ & $G A L 4 A D-E F G 1$ & $3 \cdot 2 \pm 1 \cdot 0$ & NA \\
\hline 7 & DRG1-lexA & $G A L 4 A D-E F G 1$ & $3 \cdot 9 \pm 1 \cdot 1$ & NA \\
\hline
\end{tabular}

${ }^{\star}$ Units of $\beta$-galactosidase activity $\pm \mathrm{SD}$.

$\dagger P$ value versus sample 1 determined by two-tailed $t$ test.

$\ddagger$ Strain carrying the empty vector plasmid.

$\S$ Fusions that are not expected to interact with either Drglp or Efglp.

of Drglp on invasive filamentation may be mediated through binding of Efglp.

If Drglp binds and regulates Efglp, then the defect in invasive filamentation caused by deletion of DRG1 would not be observed in the absence of Efglp. To test this hypothesis, a $\operatorname{drg} 1$ efg1 double null mutant was constructed. In contrast to a $\operatorname{drg} 1$ null mutant, which was delayed in filamentation under embedded conditions (Fig. 3), an efg1 null mutant was hyperfilamentous (Fig. 6; Giusani et al., 2002). For these experiments, the samples were incubated at room temperature, rather than at $30^{\circ} \mathrm{C}$. The lower temperature resulted in slower development of filamentous colonies, allowing detection of the hyperfilamentous phenotype. The efg1 drg1 double null mutant was also hyperfilamentous, and was indistinguishable from the efg1 single null mutant (Fig. 6). Reintroduction of the $E F G 1^{+}$ gene into either the efg1 $d r g 1$ double null mutant or the efg1 single null mutant resulted in partial complementation of the efg1 mutation, and reduced filamentation (Fig. 6;
Giusani et al., 2002). Therefore, invasive filamentation can occur in the absence of Drglp, and the effects of the $\operatorname{drg} 1$ mutation are not observed in the absence of Efglp. These results suggest that Drg1p plays a role in regulating the function of Efglp.

\section{DISCUSSION}

In this communication, we demonstrate that cells lacking the highly conserved G protein Drglp exhibit reduced invasiveness, both during growth in the laboratory and within a host. The findings that mice infected with the $\operatorname{drg} 1$ null mutant survived longer, and that growth of mutant $C$. albicans cells in the mouse kidney was reduced at an early time point, indicate that the ability of $C$. albicans to grow invasively in tissue contributes to the progression of lethal infection. Previous studies have similarly linked tissue invasiveness and virulence (e.g. Felk et al., 2002).

$D R G 1$ is a highly conserved gene with orthologues in the genomes of species from archaea to humans. The $S$. cerevisiae orthologue $\mathrm{Rbg} 1 \mathrm{p} / \mathrm{Fun} 11 \mathrm{p}$ has been shown to bind to a polysome-associated protein (Fleischer et al., 2006). C. albicans Drglp may similarly interact with polysome-associated proteins, as well as with other proteins.

Mutants lacking Drg1p are capable of forming filaments under a wide range of conditions, demonstrating that Drglp is not strictly needed for formation of filamentous cells. However, the mutant strain was defective in responding to the cues that normally promote filamentation when the organism is growing embedded within agar media. These results suggest that $\operatorname{Drg} 1 \mathrm{p}$ is involved in the mechanism for sensing or responding to signals arising from growth within agar media. One of the important cues that fungal cells experience during growth within agar is contact (Kumamoto, 2005). Therefore, since G proteins typically function as molecular switches, with active and inactive forms, the activation state of Drglp may change when cells sense contact with agar media or with host tissue, and Drglp

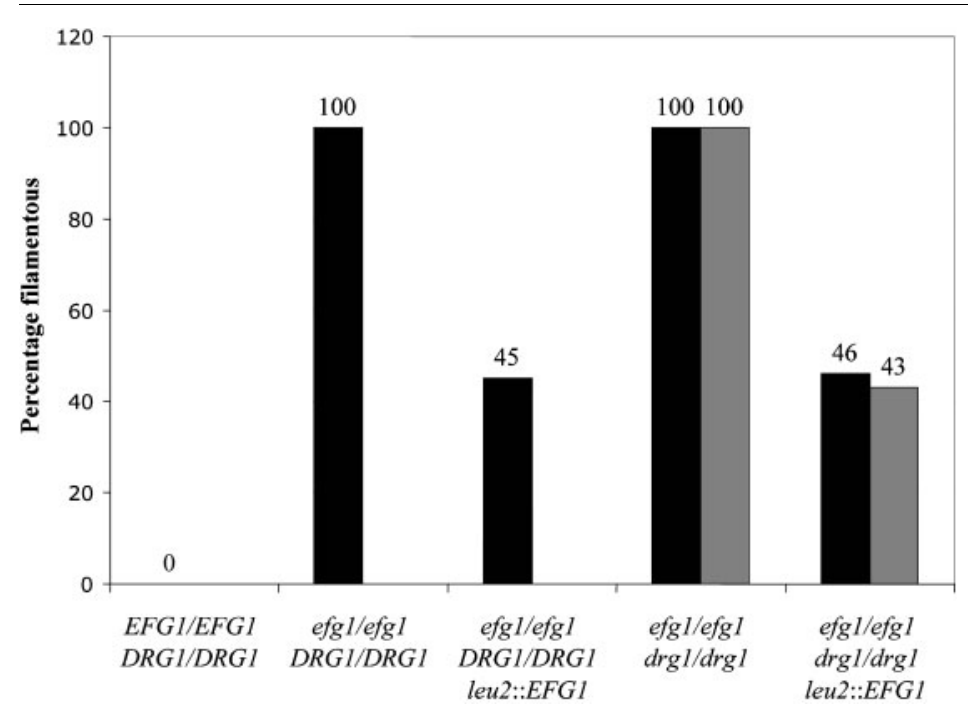

Fig. 6. Bypass of the $d r g 1$ mutant defect by deletion of EFG1. Cells were embedded in agar medium, and incubated at room temperature. After 2 days, 100-200 colonies were observed and scored for filamentation. Graph shows percentage filamentous colonies for the following strains: WT, XC121; efg 1 null, CKY136; efg1/EFG1 complemented, HLC74; drg1 efg1 double null, XC310 (two isolates analysed); and $d r g 1$ efg1/ EFG1, XC311 (two isolates analysed). Two different isolates of each strain were analyzed; the result obtained with one isolate is shown in grey and the other in black. 
may participate in contact-dependent signalling or responses.

In mammalian cells, Drglp has been previously implicated in processes related to growth control and oncogenic transformation. For example, DRG1 expression is associated with proliferating cells. In the mouse, DRG1 is relatively highly expressed in embryonic cells, and has lower expression in cells after birth (Sazuka et al., 1992). In plants, DRG is more highly expressed in growing cells as opposed to non-growing cells (Devitt et al., 1999). These findings suggest that DRG1 plays a role in a process that is necessary in proliferating cells.

In addition, ectopic expression of mouse DRG1 enhances transformation of rat embryonic fibroblasts by c-myc and ras (Mahajan et al., 1996). Since insensitivity to contactdependent regulation of cellular proliferation is a characteristic of cancer cells, ectopic expression of DRG1 may contribute to the dysregulation of this normal control mechanism, enhancing transformation. Therefore, by analogy with the function of Drg1p in C. albicans, DRG1 in mammalian cells may play a role in contact-dependent signalling, or in the response of mammalian cells to contactdependent cues.

Previous studies have demonstrated binding of mouse or human DRG proteins to the transcription factor oncogene TAL1/SCL1. This binding is mediated through the HLH region of TAL1 and the C-terminal region of DRG (Mahajan et al., 1996). C. albicans Drglp exhibited a two-hybrid interaction with the bHLH transcription factor Efglp, an important negative regulator of filamentation under embedded conditions. Since deletion of DRG1 resulted in delayed filamentation under embedded conditions, binding of Efglp by Drg1p may be a mechanism that contributes to relief of negative regulation by Efglp. Consistent with this possibility, deletion of EFG1 bypassed the defect in filamentation caused by deletion of DRG1.

Efg1p function appears to be tightly regulated through numerous mechanisms. At the transcriptional level, expression of the EFG1 gene is negatively autoregulated through binding of Efglp to the TATA box region of its promoter (Tebarth et al., 2003). At the protein level, Efglp is activated by phosphorylation by protein kinase A (Bockmuhl \& Ernst, 2001). In addition, although Efg1p is required for hyphal development in liquid medium, once hypha formation is initiated, down-regulation of EFG1 expression is observed, and forced expression of EFG1 during hyphal growth inhibits hyphal development (Tebarth et al., 2003). The findings described in this communication reveal another possible mechanism for regulation of Efglp: binding by Drglp. Drglp is cytoplasmically localized and, therefore, may regulate Efglp by affecting its localization. Many transcription factors exhibit cytoplasmic localization under certain conditions, and Efglp may undergo Drg1pdependent cytoplasmic localization when its activity needs to be inhibited, such as during embedded filamentation.
Taken together, these results suggest that tight control of Efglp function at both the transcriptional and posttranscriptional levels is important for filamentation.

In addition to filamentation, Efglp regulates expression of classical virulence factors (e.g. proteases and adhesins), as well as numerous genes involved in metabolism (Doedt et al., 2004; Nantel et al., 2002). Thus, the role of Efglp in cellular physiology goes well beyond its role in controlling cellular morphology. In this context, the tight regulation of Efglp function through multiple regulatory mechanisms allows careful control of a wide range of cellular processes that are impacted by this important transcription factor.

\section{ACKNOWLEDGEMENTS}

We thank Dr Lauren Richey for assistance with histology, Paola Zucchi for immuofluorescence microscopy and photography, and Sarah Jane White for technical assistance. We are also grateful to Drs Ralph Isberg, Dean Dawson, Claire Moore, Perry Riggle, Marcelo Vinces and Igor Bruzual for helpful discussions, and to Dr Andrew Wright for helpful discussions and comments on the manuscript. This research was supported by NIH grant AI038591 from the National Institute of Allergy and Infectious Diseases (to C. A. K.).

\section{REFERENCES}

Ausubel, F., Brent, R., Kingston, R., Moore, D., Seidman, J., Smith, J. \& Struhl, K. (1989). Current Protocols in Molecular Biology. New York: Wiley.

Bockmuhl, D. P. \& Ernst, J. F. (2001). A potential phosphorylation site for an A-type kinase in the Efg1 regulator protein contributes to hyphal morphogenesis of Candida albicans. Genetics 157, 1523-1530.

Brand, A., MacCallum, D. M., Brown, A. J., Gow, N. A. \& Odds, F. C. (2004). Ectopic expression of URA3 can influence the virulence phenotypes and proteome of Candida albicans but can be overcome by targeted reintegration of URA3 at the RPS10 locus. Eukaryot Cell 3, 900-909.

Brown, D. H., Jr, Slobodkin, I. V. \& Kumamoto, C. A. (1996). Stable transformation and regulated expression of an inducible reporter construct in Candida albicans using restriction enzyme-mediated integration. Mol Gen Genet 251, 75-80.

Brown, D. H., Jr, Giusani, A. D., Chen, X. \& Kumamoto, C. A. (1999). Filamentous growth of Candida albicans in response to physical environmental cues and its regulation by the unique $C Z F 1$ gene. $M o l$ Microbiol 34, 651-662.

Burke, D., Dawson, D. \& Stearns, T. (2000). Methods in Yeast Genetics. Cold Spring Harbor, NY: Cold Spring Harbor Laboratory.

Chen, X., Magee, B. B., Dawson, D., Magee, P. T. \& Kumamoto, C. A. (2004). Chromosome 1 trisomy compromises the virulence of Candida albicans. Mol Microbiol 51, 551-565.

Clontech (2001). Clontech Yeast Protocols Handbook. Manual no. PT3024-1. Palo Alto, CA: Clontech Laboratories.

Devitt, M. L., Maas, K. J. \& Stafstrom, J. P. (1999). Characterization of DRGs, developmentally regulated GTP-binding proteins, from pea and Arabidopsis. Plant Mol Biol 39, 75-82.

Doedt, T., Krishnamurthy, S., Bockmuhl, D. P., Tebarth, B., Stempel, C., Russell, C. L., Brown, A. J. \& Ernst, J. F. (2004). 
APSES proteins regulate morphogenesis and metabolism in Candida albicans. Mol Biol Cell 15, 3167-3180.

Felk, A., Kretschmar, M., Albrecht, A. \& 7 other authors (2002). Candida albicans hyphal formation and the expression of the Efg1regulated proteinases Sap4 to Sap6 are required for the invasion of parenchymal organs. Infect Immun 70, 3689-3700.

Fleischer, T. C., Weaver, C. M., McAfee, K. J., Jennings, J. L. \& Link, A. J. (2006). Systematic identification and functional screens of uncharacterized proteins associated with eukaryotic ribosomal complexes. Genes Dev 20, 1294-1307.

Fonzi, W. A. \& Irwin, M. Y. (1993). Isogenic strain construction and gene mapping in Candida albicans. Genetics 134, 717-728.

Giusani, A. D., Vinces, M. \& Kumamoto, C. A. (2002). Invasive filamentous growth of Candida albicans is promoted by Czflp-dependent relief of Efglp-mediated repression. Genetics 160, 1749-1753.

Golemis, E. A., Gyuris, J. \& Brent, R. (1996). Interaction trap/twohybrid system to identify interacting proteins. In Current Protocols in Molecular Biology. Edited by F. Ausubel, R. Brent, R. Kingston, D. Moore, J. Seidman, J. Smith \& K. Struhl. New York: Wiley.

James, P., Halladay, J. \& Craig, E. A. (1996). Genomic libraries and a host strain designed for highly efficient two-hybrid selection in yeast. Genetics 144, 1425-1436.

Kelly, R., Miller, S. M., Kurtz, M. B. \& Kirsch, D. R. (1987). Directed mutagenesis in Candida albicans: one-step gene disruption to isolate ura3 mutants. Mol Cell Biol 7, 199-208.

Kumamoto, C. A. (2005). A contact-activated kinase signals Candida albicans invasive growth and biofilm development. Proc Natl Acad Sci U S A 102, 5576-5581.

Kumamoto, C. A. \& Vinces, M. D. (2005a). Alternative Candida albicans lifestyles: growth on surfaces. Annu Rev Microbiol 59, 113-133.

Kumamoto, C. A. \& Vinces, M. D. (2005b). Contributions of hyphae and hypha-co-regulated genes to Candida albicans virulence. Cell Microbiol 7, 1546-1554.

Kumar, S., Iwao, M., Yamagishi, T., Noda, M. \& Asashima, M. (1993). Expression of GTP-binding protein gene drg during Xenopus laevis development. Int J Dev Biol 37, 539-546.

Lay, J., Henry, L. K., Clifford, J., Koltin, Y., Bulawa, C. E. \& Becker, J. M. (1998). Altered expression of selectable marker URA3 in genedisrupted Candida albicans strains complicates interpretation of virulence studies. Infect Immun 66, 5301-5306.
Lo, H. J., Kohler, J. R., DiDomenico, B., Loebenberg, D., Cacciapuoti, A. \& Fink, G. R. (1997). Nonfilamentous C. albicans mutants are avirulent. Cell 90, 939-949.

Longtine, M. S., McKenzie, A., 3rd, Demarini, D. J., Shah, N. G., Wach, A., Brachat, A., Philippsen, P. \& Pringle, J. R. (1998). Additional modules for versatile and economical PCR-based gene deletion and modification in Saccharomyces cerevisiae. Yeast 14, 953-961.

Mahajan, M. A., Park, S. T. \& Sun, X. H. (1996). Association of a novel GTP binding protein, DRG, with TAL oncogenic proteins. Oncogene 12, 2343-2350.

Miller, J. H. (1972). Experiments in Molecular Genetics. Cold Spring Harbor, NY: Cold Spring Harbor Laboratory.

Nantel, A., Dignard, D., Bachewich, C. \& 12 other authors (2002). Transcription profiling of Candida albicans cells undergoing the yeast-to-hyphal transition. Mol Biol Cell 13, 3452-3465.

Pringle, J. R., Adams, A. E., Drubin, D. G. \& Haarer, B. K. (1991). Immunofluorescence methods for yeast. Methods Enzymol 194, 565-602.

Sambrook, J., Fritsch, E. F. \& Maniatis, T. (1989). Molecular Cloning: a Laboratory Manual, 2nd edn. Cold Spring Harbor, NY: Cold Spring Harbor Laboratory

Sazuka, T., Tomooka, Y., Ikawa, Y., Noda, M. \& Kumar, S. (1992). DRG: a novel developmentally regulated GTP-binding protein. Biochem Biophys Res Commun 189, 363-370.

Schenker, T., Lach, C., Kessler, B., Calderara, S. \& Trueb, B. (1994). A novel GTP-binding protein which is selectively repressed in SV40 transformed fibroblasts. J Biol Chem 269, 25447-25453.

Sommer, K. A., Petersen, G. \& Bautz, E. K. (1994). The gene upstream of DmRP128 codes for a novel GTP-binding protein of Drosophila melanogaster. Mol Gen Genet 242, 391-398.

Stoldt, V. R., Sonneborn, A., Leuker, C. E. \& Ernst, J. F. (1997). Efglp, an essential regulator of morphogenesis of the human pathogen Candida albicans, is a member of a conserved class of bHLH proteins regulating morphogenetic processes in fungi. $E M B O$ J 16, 1982-1991.

Tebarth, B., Doedt, T., Krishnamurthy, S., Weide, M., Monterola, F., Dominguez, A. \& Ernst, J. F. (2003). Adaptation of the Efglp morphogenetic pathway in Candida albicans by negative autoregulation and PKA-dependent repression of the EFG1 gene. J Mol Biol 329, 949-962. 